\title{
The lipoxygenase gene family: a genomic fossil of shared polyploidy between Glycine max and Medicago truncatula Jin Hee Shin ${ }^{1}$, Kyujung Van ${ }^{1}$, Dong Hyun Kim¹, Kyung Do Kim¹, Young Eun Jang1, Beom-Soon Choi ${ }^{2}$, Moon Young Kim ${ }^{1,3}$ and Suk-Ha Lee*1,2,3,4
}

Address: ${ }^{1}$ Department of Plant Science, Seoul National University, Seoul 151-921, Korea, ${ }^{2}$ National Instrumentation Center for Environmental Management, Seoul National University, Seoul 151-921, Korea, ${ }^{3}$ Research Institute for Agriculture and Life Sciences, Seoul National University, Seoul 151-921, Korea and ${ }^{4}$ Plant Genomic and Breeding Research Institute, Seoul National University, Seoul, 151-921, Korea

Email: Jin Hee Shin - kaloseidos@hotmail.com; Kyujung Van - kvan@snu.ac.kr; Dong Hyun Kim - andronic@snu.ac.kr; Kyung Do Kim - kdkim01@snu.ac.kr; Young Eun Jang - j.easttree@gmail.com; Beom-Soon Choi - bschoi@nicem.snu.ac.kr; Moon Young Kim - moonykim@snu.ac.kr; Suk-Ha Lee* - sukhalee@snu.ac.kr

* Corresponding author

Published: 23 December 2008

BMC Plant Biology 2008, 8:133
Received: 8 August 2008

Accepted: 23 December 2008

This article is available from: http://www.biomedcentral.com//47/-2229/8//33

(C) 2008 Shin et al; licensee BioMed Central Ltd.

This is an Open Access article distributed under the terms of the Creative Commons Attribution License (http://creativecommons.org/licenses/by/2.0), which permits unrestricted use, distribution, and reproduction in any medium, provided the original work is properly cited.

\begin{abstract}
Background: Soybean lipoxygenases (Lxs) play important roles in plant resistance and in conferring the distinct bean flavor. Lxs comprise a multi-gene family that includes $G m L x I, G m L x 2$ and $G m L x 3$, and many of these genes have been characterized. We were interested in investigating the relationship between the soybean lipoxygenase isozymes from an evolutionary perspective, since soybean has undergone two rounds of polyploidy. Here we report the tetrad genome structure of soybean $L x$ regions produced by ancient and recent polyploidy. Also, comparative genomics with Medicago truncatula was performed to estimate $L x s$ in the common ancestor of soybean and Medicago.

Results: Two $L x$ regions in Medicago truncatula showing synteny with soybean were analyzed. Differential evolutionary rates between soybean and Medicago were observed and the median $\mathrm{Ks}$ values of $\mathrm{Mt}-\mathrm{Mt}, \mathrm{Gm}$ $\mathrm{Mt}$, and $\mathrm{Gm}-\mathrm{Gm}$ paralogs were determined to be $0.75,0.62$, and 0.46 , respectively. Thus the comparison of $\mathrm{Gm}-\mathrm{Mt}$ paralogs $(\mathrm{Ks}=0.62)$ and $\mathrm{Gm}-\mathrm{Mt}$ orthologs $(\mathrm{Ks}=0.45)$ supports the ancient duplication of $L x$ regions in the common ancestor prior to the Medicago-Glycine split. After speciation, no $L x$ regions generated by another polyploidy were identified in Medicago. Instead tandem duplication of $L x$ genes was observed. On the other hand, a lineage-specific duplication occurred in soybean resulting in two pairs of $L x$ regions. Each pair of soybean regions was co-orthologous to one $L x$ region in Medicago. A total of 34 $L x$ genes ( $15 \mathrm{MtLxs}$ and $19 \mathrm{GmLxs}$ ) were divided into two groups by phylogenetic analysis. Our study shows that the $L x$ gene family evolved from two distinct $L x$ genes in the most recent common ancestor.

Conclusion: This study analyzed two pairs of $L x$ regions generated by two rounds of polyploidy in soybean. Each pair of soybean homeologous regions is co-orthologous to one region of Medicago, demonstrating the quartet structure of the soybean genome. Differential evolutionary rates between soybean and Medicago were observed; thus optimized rates of Ks per year should be applied for accurate estimation of coalescence times to each case of comparison: soybean-soybean, soybean-Medicago, or Medicago-Medicago. In conclusion, the soybean $L x$ gene family expanded by ancient polyploidy prior to taxon divergence, followed by a soybean- specific duplication and tandem duplications, respectively.
\end{abstract}




\section{Background}

Lipoxygenases (LOXs) have been intensively studied for the past century and have been reported in yeast, algae, fungi, animals, and plants [1]. In higher plants, LOXs are almost ubiquitous and involved in various physiological processes. Importantly, the oxidized products by these enzymes are involved in the traumatic acid and jasmonic acid (JA) pathways, which confer various biotic and abiotic resistance traits to plants [2-4]. Soybean lipoxygenases have received significant attention, since the oxidized compounds cause soy products to have an unpleasant flavor $[1,5,6]$. Many lipoxygenase isozymes have been isolated and are well characterized, but these studies were mainly focused on LOX 1, 2 and 3, the lipoxygenases preferentially expressed in seeds. Moreover, an analysis of soybean mutant lines lacking the isozymes has shown that the $L x 1$ and $L x 2$ loci are tightly linked, while the $L x 3$ locus is independent of the other two loci $[7,8]$. Additionally, several vegetative $L x$ genes, such as $L x 4, L x 5, L x 6, L x 7$, and $L x 8$, have been detected and characterized $[9,10]$.

A number of $L x$ loci have been identified in the fully sequenced Arabidopsis thaliana and almost completed Medicago genomes. This suggests that there are more $L x$ genes not yet reported in the soybean genome, and that the number of soybean Lxs would outnumber those in Arabidopsis and Medicago. The expansion of a gene family is related to gene duplication events, such as whole genome duplication, tandem duplication, and transposition [11]. An investigation of the $L x$ gene family expansion is also of interest in the context of soybean genome evolution, since soybean is known to have undergone two rounds of polyploidy events by analyses of ESTs $[12,13]$. The duplicated soybean genome has been investigated by RFLP mapping and more than two regions have been detected by RFLP probes [14]. Furthermore, analyses of homeologous BAC clones, anchored by FAD2 and HCBT genes, revealed highly conserved regions produced by a recent duplication 14.5 million years ago (MYA) $[15,16]$. The presence of duplicated soybean chromosomal regions was substantiated by analyzing seventeen homeologous BACs [17]. These studies also showed the genome rearrangement of homeologous regions after whole genome duplication.

Genome duplication events are common in most crop plants [18]. Even model plants, such as Arabidopsis and Medicago, have undergone at least one round of genome doubling $[12,19]$. A comparative genomic approach using Medicago has provided insights into complex legume genomes, which cannot be satisfactorily studied provided by the model plant $A$. thaliana [20]. The divergence of soybean and Medicago from a common ancestor which experienced genome duplication was estimated at 50 MYA [14]. Medicago and soybean, two closely related legume plants, have had two bursts of gene duplication, but it is not clear whether they had a shared polyploidy event before taxon divergence. To clarify this issue, a phylogenetic analysis of gene families was performed, and a hypothesis of shared polyploidy prevailed over the alternative hypothesis of taxon divergence prior to duplication [21]. Later, the early duplication of soybean before the Medicago-soybean split was supported by the whole genome duplication which predated speciation between Medicago and Lotus japonicus [22].

With regard to genome conservation in legumes, many studies have shown broad-scale conservation of legume genomes and gene order $[23,24]$. Not only among soybean, Medicago and Arabidopsis [24] but microsynteny was also observed among three genomes [25]. Mudge et al. (2005) [26] identified very high synteny between $3 \mathrm{Mb}$ of soybean DNA sequences and 2 Medicago chromosomes. A recent study also demonstrated a network of synteny within conserved regions among Arabidopsis, Medicago and soybean [27].

In this study the evolutionary expansion of the soybean $L x$ gene family was demonstrated to occur by two rounds of polyploidy and the evolutionary relationships of nineteen $L x$ genes in four homeologous chromosomal regions were explored. Moreover, the differential rates of evolution in orthologous and paralogous regions of the $L x$ gene regions between soybean and Medicago reflect the history of the paleopolyploid soybean genome.

\section{Results}

Assembly and Mapping of Soybean BACs and Scaffolds

PCR-based screens of the gmw1 BAC library identified a total of six BAC clones: gmw1-45b2 and gmw1-91g6 for both $L x 1$ and $L x 2$; gmw1-6b18, gmw1-9c4, gmw1-22a20, and gmw1-22f19 for $L x 3$. In other words, $L x 1$ and $L x 2$ are located on the same BACs, but $L x 3$ is on different BACs, which is in accordance with many previous reports $[8,28]$. The six BAC clones were sequenced using 454 sequencing technology and the average read length was $250 \mathrm{bp}$. The number of contigs varied from 1 to 25 and the largest assembled contig was $36 \mathrm{~kb}$ (see Additional file 1). Two BAC clones, gmw1-22a20 and gmw1-22f19, were fully sequenced. The remaining gaps were closed by hybridization assemblies, adding ABI-Sanger sequences amplified across the gaps [29]. Here, we mainly used gmw1-9c4 containing $L x 3$ and gmw1-91g 6 containing $L x 1$ and $L x 2$ for further analysis (Figure 1). More than ten scaffolds containing lipoxygenase genes were identified from the $7 \times$ whole genome sequencing (WGS) assembly from early 2008 http://www.phytozome.com/soybean and selected scaffolds showing synteny with BAC clones were analyzed for further study. Sequences of gmw1-9c4 and gmw1$91 \mathrm{~g} 6$ were embedded in Scaffold 88 and Scaffold 134, 


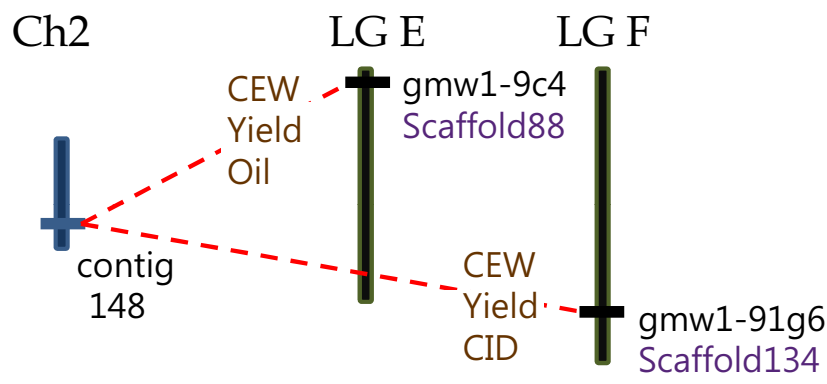

\section{M.truncatula G.max}

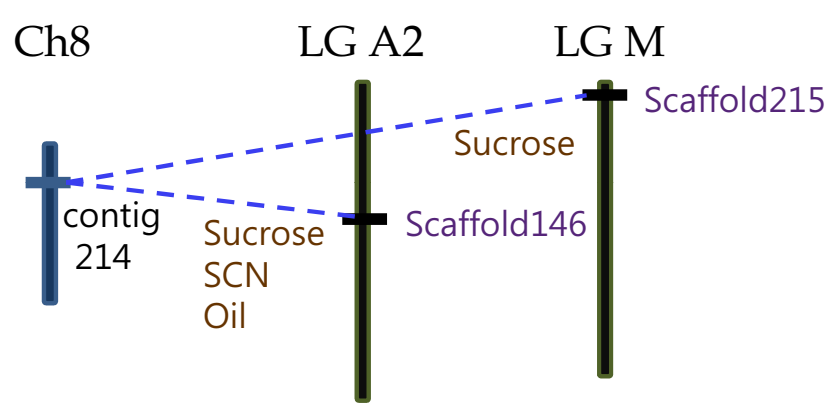

Figure I

Comparative map of six $L x$ regions from soybean and Medicago. In silico genetic mapping with SSR markers placed the soybean $L x$ regions into four different linkage groups (LGs). Two BAC clones, gmwl-9c4 and gmwl-9lg6, were selected containing $L x I, L x 2$ and $L x 3$, and these genes were embedded in Scaffolds 88 and I34. Two blocks showing synteny with selected BACs were detected in Medicago and contig I 48 on Medicago chromosome 2 was more similar to the BACs. Blast search with the sequence of contig 214 on Medicago chromosome 8 resulted in two more soybean $L x$ scaffolds. Soybean QTLs for each $L x$ region are denoted as brown characters and some of them are conserved among the four regions.

respectively. Also, the sequences of Scaffold 146 and Scaffold 215 were highly identical to each other and showed colinearity with those BAC clones (Figures 1 and 2). Scaffold 88, Scaffold 134, Scaffold 146, and Scaffold 215 were named $\mathrm{GmA}, \mathrm{GmA}^{\prime}, \mathrm{GmB}$, and $\mathrm{GmB}^{\prime}$, respectively. A total of 13 soybean $L x$ genes were searched on NCBI and nine of them were included in $\mathrm{GmA}, \mathrm{GmA}^{\prime}, \mathrm{GmB}$, and $\mathrm{GmB}^{\prime}$. Two of the soybean $L x$ genes did not have proper scaffolds with high scores, and the scaffold containing GmLOX9 did not show any synteny except for the GmLOX9 gene itself (see Additional file 2).

Genetic mapping was achieved by identification of lipoxygenase genes and simple sequence repeat (SSR) markers placed on the composite map http://www.soybase.org. Previously, GmLx1 and GmLx2 on Scaffold 134 were mapped to linkage group (LG) F and GmLx3 on Scaffold 88 was mapped to LG E $[7,28,30]$. By in silico mapping

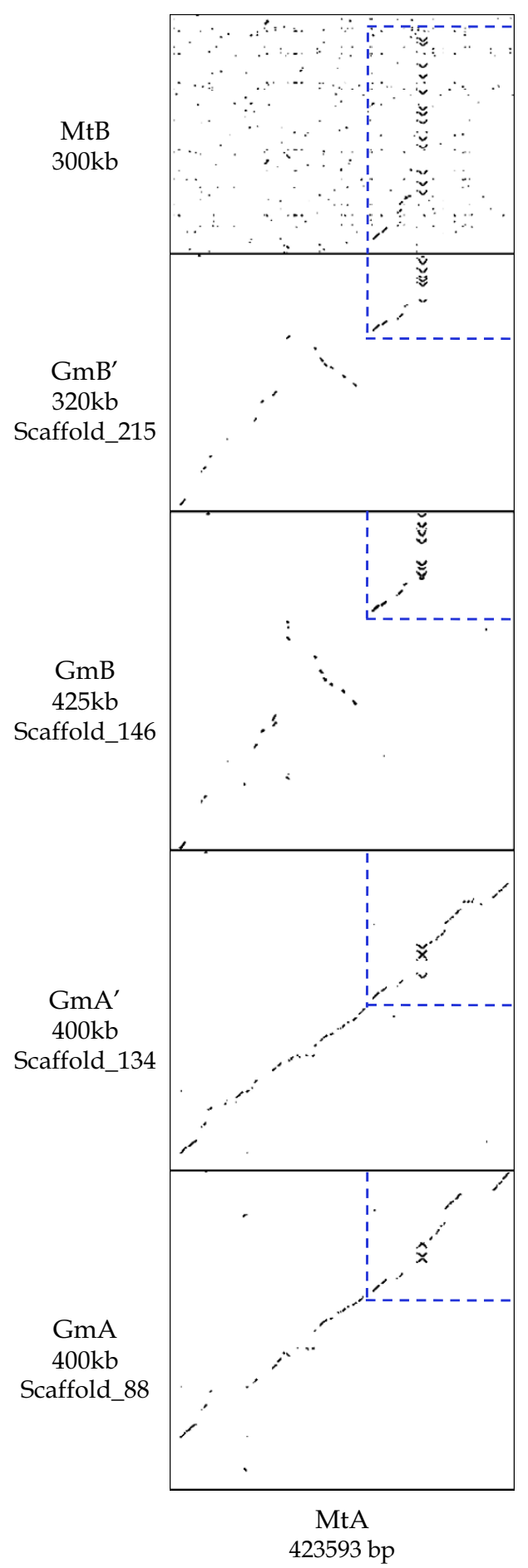

Figure 2

Dot plot alignments of six $L x$ regions between soybean and Medicago. MtA shows a high level of similarity with $\mathrm{GmA}$ and $\mathrm{GmA}$ '. Both $\mathrm{GmB}$ and $\mathrm{GmB}$ ' have an inversion block, and their sequences end with repetitive lipoxygenases like MtB. Common sequences among the six regions are highlighted with blue dotted lines. 
based on sequence, the four scaffolds were placed on four different LGs: Scaffold 88 was anchored by Satt575, Satt213, Sat_112, and Satt411 on LG E; Scaffold 134 contained Sat_090, Satt656, and Sat_417 on LG F; Scaffold 146 had Sat_115, Sat_199, Sat_129, Sat_233, and Satt089 on LG A2; Scaffold 215 was mapped to LG M by Sat_389, Satt404 and Sat_391.

Numerous QTLs have been related to these four $L x$ regions in soybean and some of them have been associated with more than one region: corn earworm resistance (CEW) and yield QTLs on part of LG E and LG F [31-34]; sucrose content QTLs on LG A2 and LG M [35], oil QTLs on LG E and LG A2 [36,37]. These mutually conserved QTLs indicate that specific genes associated with CEW, yield, sucrose, and oil have been retained across homeologous genomic regions after genome duplication (Figure 1). Additionally, the carbon isotope discrimination (CID) on LG F and soybean cyst nematode resistance (SCN) on LG A2 have been reported $[32,38]$.

\section{Comparison of Lx Regions in G. max and M. truncatula}

Two $L x$ regions colinear to these two soybean BACs were detected on Medicago chromosomes 2 and 8 in Medicago pseudomolecule $2.0 \mathrm{http}: / /$ www.medicago.org/genome and named $\mathrm{MtA}$ and $\mathrm{MtB}$, respectively (Figure 2). $\mathrm{MtA}$ consists of five BAC clones: AC148918, AC137554, AC146308, AC136955 and AC155896. MtB is comprised of four BAC clones: AC149580, AC140032, AC149638 and AC174341. A dot-plot analysis of the six $L x$ regions between soybean and Medicago revealed that all showed synteny with some genome rearrangement by insertion, deletion, and tandem duplication. MtA shared most of the genes with the two soybean BACs; however, Mt8 contig 214 showed synteny with only short regions of the both ends of the soybean BACs, with tandem duplicated $L x s$ being observed instead. Also, a search in the Medicago database http://www.tigr.org/tdb/e2k1/mta1/ identified $32 \mathrm{Lx}$ gene loci. Only $15 \mathrm{Lxs}$ in these two regions were further analyzed because the remaining loci did not show any synteny with soybean $L x$ regions.

Detailed gene structure and comparisons of the six $L x$ regions are shown by blue dotted lines (Figure 2) and BLASTZ (Figure 3). The Ks values between homologous genes were calculated (see Additional file 3). Full annotation of the genes is available in Additional file 4. A total of 15 pairs of combinations between the six regions were compared based on their Ks values (Table 1). By comparing the median Ks values of common genes among the six regions, differential evolutionary rates between Medicago and soybean were observed. The median Ks value between $\mathrm{MtA}$ and MtB was 0.75, which was close to the Medicago older peak estimated by other analyses $[12,13,22]$. The median Ks value between $\mathrm{Gm}-\mathrm{Gm}$ paralogs was similar to previous reports $[12,13]$. However, the median Ks value between Gm-Mt paralogs was smaller than Mt-Mt paralogs, but larger than Gm-Gm paralogs (Tables 1 and 2). The median Ks value of Gm-Mt orthologs was almost the same as that of $\mathrm{Gm}-\mathrm{Gm}$ paralogs. The median Ks value of GmA-GmA' and GmB-GmB' were 0.11 and 0.10 , respectively, suggesting they were produced by a recent polyploidy in soybean like the event defining the FAD2 gene family and HCBT gene regions $[15,16]$.

The gene density of the six Lx regions was similar: one gene per $7.06 \mathrm{~kb}$ in $\mathrm{MtA}$; one gene per $8.11 \mathrm{~kb}$ in $\mathrm{MtB}$; one gene per $7.27 \mathrm{~kb}$ in $\mathrm{GmA}$; one gene per $7.55 \mathrm{~kb}$ in $\mathrm{GmA}^{\prime}$; one gene per $7.59 \mathrm{~kb}$ in $\mathrm{GmB}$; one gene per $7.62 \mathrm{~kb}$ in $\mathrm{GmB}^{\prime}$. The density of these regions in Medicago was not significantly different from that of the homologous regions in soybean, consistent with previous reports of one gene per $6 \mathrm{~kb}$ or $5.8-6.7 \mathrm{~kb}[16,26,39]$. The average GC content was approximately the same among those regions: $32.68 \%$ in MtA; $32.52 \%$ in $\mathrm{MtB} ; 32.14 \%$ in $\mathrm{GmA}$; $32.05 \%$ in $\mathrm{GmA}^{\prime} ; 31.96 \%$ in $\mathrm{GmB} ; 31.17 \%$ in $\mathrm{GmB}^{\prime}$. Among the six Lx regions in this study, GmA and GmA' were more similar to $\mathrm{MtA}$, whereas $\mathrm{GmB}$ and $\mathrm{GmB}^{\prime}$ were closer to MtB (Figs. 2, 3).

\section{Phylogenetic Analysis of Lx Genes in Soybean and Medicago}

A total of $34 \mathrm{Lxs}$ were detected from the six homologous $L x$ regions: 2 in MtA; 13 in MtB; 3 in GmA; 4 in $\mathrm{GmA}^{\prime} ; 7$ in $\mathrm{GmB} ; 5$ in GmB' (Figure 3). For convenience, each $L x$ gene was named according to its species, chromosomal region, and physical order. Thus, their designated names are different from their GeneIDs in GenBank. Because the $L x$ gene structures were very similar, their evolutionary relationships were uncovered by calculating their Ks values. The Ks values among ten $L x s$ (from $M t B \_L x 2$ to $\left.M t B \_L x 11\right)$ ranged from 0.3440 to 0.6393 , indicating extensive tandem duplication of $L x$ genes after whole genome duplication in Medicago. Phylogenetic analysis using parsimony of $34 L x$ genes in the six regions classified these $34 \mathrm{Lx}$ genes into two clades denoted as black and white squares (Figure 4 ). The grouping of $L x$ genes showed that GmB Lxs were more similar to MtB Lxs than to GmA or GmA' Lxs. In other words, the divergence time between GmA and GmB was earlier than the time of speciation between the two species. After taxon divergence, $\mathrm{GmA}$ and $\mathrm{GmB}$ regions were duplicated resulting in $\mathrm{GmA}$, GmA', GmB, and GmB'. In Medicago, the tandem duplication of $L x$ genes was observed instead of another polyploidy.

\section{Discussion}

Ancient polyploidy in the Lx regions of common ancestor Previously, it had not been clear whether soybean and Medicago shared a polyploidy event because the old peak 


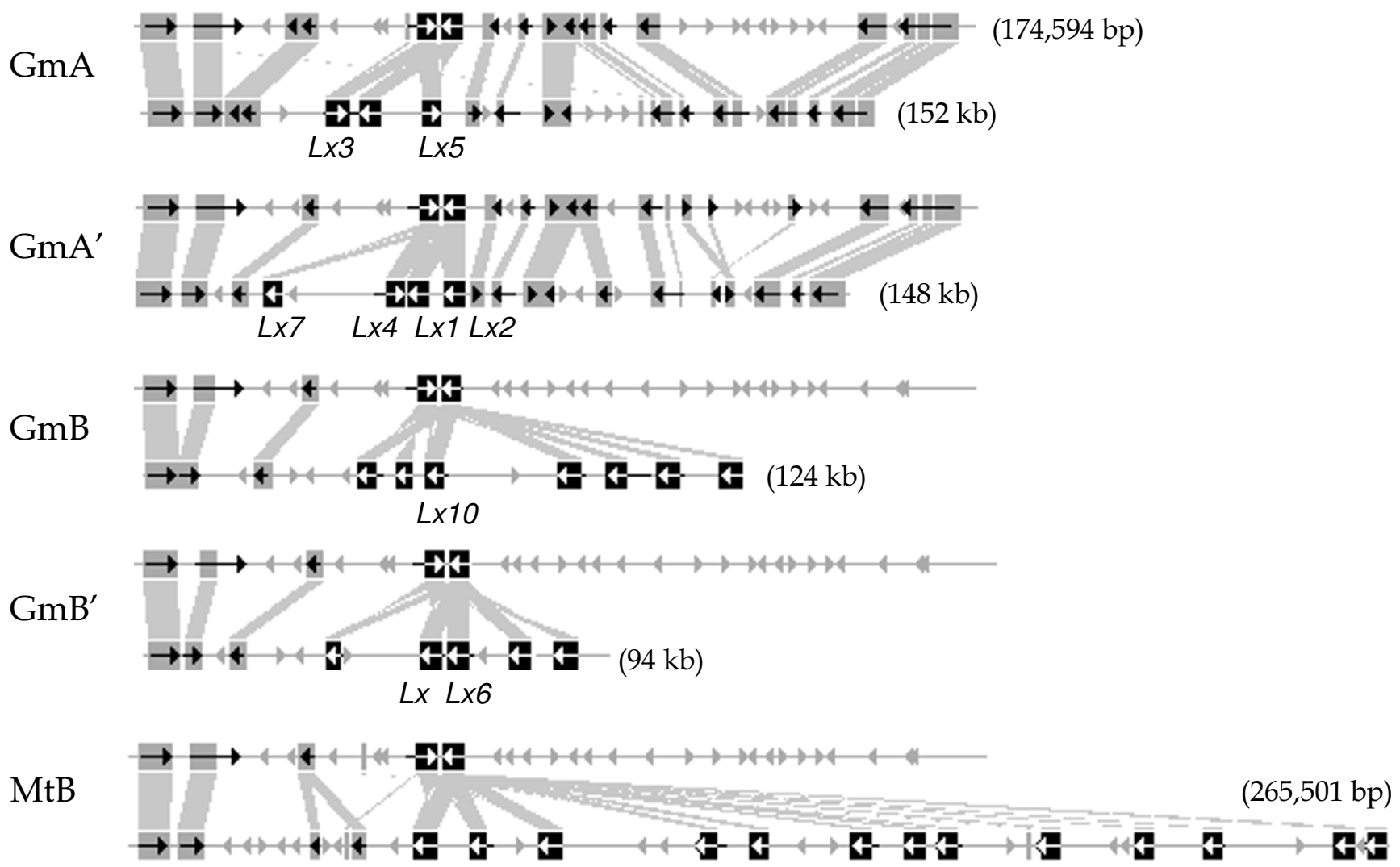

Figure 3

Diagrammatic representation of gene conservation between the six Lx regions by BLASTZ. The sequence highlighted with blue dotted lines in Figure 2 was analyzed in detail with gene prediction. The length and orientation of predicted genes are represented as arrows, and homologous sequences are connected with grey boxes. Each lipoxygenase is depicted as a black box and white arrow, and soybean $L x$ genes registered in GenBank are denoted with their gene names. A total of $34 L x$ loci, 15 from Medicago and 19 from soybean, were detected.

of paralog Ks in Medicago did not overlap with that of the soybean $[12,13]$. To explain the gap between the soybean and Medicago paralog Ks peaks, Blanc and Wolfe hypothesized that the soybean lineage split from one of the allopolyploid genomes of Medicago [12]. Later, an analysis of gene families provided a framework of shared polyploidy prior to taxon divergence [21]. A total of 56\% of gene families also supported a shared soybean-Medicago duplication before the split, whereas the remaining gene families supported alternative hypotheses, including taxon divergence prior to the ancient polyploidy [40]. In addition, a lower synonymous substitution rate in soybean was suggested to explain the difference between Medicago and soybean Ks value peaks $[13,40]$.

Our data corroborates the hypothesis that the two peaks of median Ks values in soybean and Medicago actually represent the same event but show differential synonymous substitution rates. While orthologs refer to homologous genes that have been generated by speciation, paralogs are homologous genes generated via duplication [41]. The median Ks values of Mt-Mt paralogs, Gm-Mt paralogs, and Gm-Gm paralogs revealed the differential evolutionary rates between the two species (Table 1). The median Ks value of Mt-Mt paralogs $(\mathrm{Ks}=0.75)$ is greater than that of Gm-Gm paralogs ( $\mathrm{Ks}=0.46$ ), while the $\mathrm{Gm}-\mathrm{Mt}$ value is intermediate. Thus, to decide the chronological order of duplication and taxon divergence without bias produced by differential evolutionary rates, it is absolutely crucial to compare the values within the same category. A comparison of Gm-Mt paralogs ( Ks = 0.62) and Gm-Mt orthologs $(\mathrm{Ks}=0.45)$ indicates that ancient duplication occurred prior to speciation (Table 2). In conclusion, the Mt-Mt, Gm-Mt and Gm-Gm paralogs actually represent the same duplication event, although their absolute values look different.

Recently, a large-scale duplication between Medicago and L. japonicus was proven to have occurred before speciation [22]. The Ks distribution of ancient duplication between Medicago and Lotus was not significantly different, even though Medicago had a narrower peak and Lotus showed a 
Table I: Median Ks values for combinations of pairs between six Lx regions from Medicago and soybean

\begin{tabular}{cc}
\hline Combinations of pairs & Median Ks \\
\hline Ancient polyploidy & \\
Mt-Mt paralog & \\
MtA-MtB & 0.75 \\
Gm-Mt paralog & \\
MtA-GmB & 0.58 \\
MtA-GmB' & 0.60 \\
MtB-GmA & 0.64 \\
MtB-GmA' & 0.67 \\
Gm-Gm paralog & \\
GmA-GmB & 0.46 \\
GmA-GmB' & 0.45 \\
GmA'-GmB & 0.48 \\
GmA'-GmB' & 0.46 \\
Taxon divergence & \\
Gm-Mt ortholog & \\
MtA-GmA & 0.40 \\
MtA-GmA' & 0.41 \\
MtB-GmB & 0.49 \\
MtB-GmB' & 0.50 \\
Recent polyploidy & \\
Gm-Gm paralog & \\
GmA-GmA' & 0.11 \\
GmB-GmB' & 0.10 \\
\hline
\end{tabular}

broader peak. The median Ks value of older polyploidy in Medicago and Lotus had been estimated to be 0.7 to 0.9 $[12,13,22]$. In our study, the Ks value of older polyploidy in soybean was much smaller, consistent with previous studies (Table 2) $[12,13,21]$. Thus, optimized rates of Ks per year should be applied for balanced estimation of coalescence times to each case of comparison: soybean-soybean, soybean-Medicago or Medicago-Medicago.

Most crop legumes belong to the Hologalegina and phaseoloid-millettioid clades [42]. The earlier duplication between Medicago and Lotus is the duplication event in the common ancestor of the Hologalegina clade, which includes Medicago, Lotus, and Pisum. Soybean belongs to the phaseoloid-millettioid clade, which contains Glycine, Phaseolus, and Vigna. Taken together, our data support an ancient duplication event in the common ancestor of the Hologalegina and phaseoloid-millettioid clades.

\section{Evolutionary change of soybean and Medicago after speciation}

It has been suggested that the younger peak in Medicago did not correspond to another polyploidy but a series of tandem duplications because the peak was too broad $[21,40]$. Also, there was no clear Ks peak suggesting large scale duplication after the Medicago-Lotus split [22]. In this study, only two Medicago $L x$ regions produced by ancient polyploidy were detected, and no chromosomal region generated by recent duplication was identified. Instead, ten occurrences of extensive single gene duplication were observed in one Medicago $L x$ region. The colinearity between MtA and MtB was not high except for repetitive Lxs and a few flanking genes (Figure 2). It is thought that these duplicated regions were differentiated by a diploidization process.

A total of four soybean chromosomal regions were anchored by three to seven $L x$ genes. Among the four $L x$ regions, the level of similarity and sequence conservation was high between regions produced by the recent duplication (Figures. 2, 3). These two pairs of $L x$ regions were generated by two rounds of polyploidy in soybean. With respect to the conservation level of sequence and structure, both inter- and intra-pairs showed synteny (e.g. GmA-GmB, GmA-GmB', GmA'-GmB, and GmA'-GmB'). The level of diploidization in soybean $L x$ regions generated by ancient polyploidy was lower than that of Medicago. Moreover, the conserved QTLs among the four regions- sucrose, oil, yield, and corn earworm resistancesupport their duplicated origin (Figure 1).

Until now, sequence-based analyses of the soybean genome have been focused on regions produced by recent polyploidy $[15-17,43]$. The comparative genomics approach used in this paper furthered our understanding of the soybean genome and allowed us to speculate on chromosomal regions produced by both recent and ancient duplication events. Furthermore, each pair of $L x$ regions in soybean was close to an $L x$ region in Medicago. Co-orthologs refer to genes generated by a lineage-specific duplication [41]. Thus, $\mathrm{GmA} / \mathrm{GmA}^{\prime}$ and $\mathrm{GmB} / \mathrm{GmB}^{\prime}$ are the co-orthologous chromosomal regions to MtA and $\mathrm{MtB}$, respectively (Figure 1 ). In this case, it is difficult to

Table 2: Ks estimations of ancient polyploidy and taxon divergence

\begin{tabular}{|c|c|c|c|c|c|}
\hline Materials & Mt-Mt paralogs ${ }^{\mathrm{a}}$ & Gm-Mt paralogs ${ }^{a}$ & Gm-Gm paralogs ${ }^{a}$ & Gm-Mt orthologs ${ }^{b}$ & References \\
\hline ESTs & $0.65-0.70$ & - & $0.45-0.50$ & $0.40-0.50$ & Blanc and Wolfe, 2004 \\
\hline ESTs & 0.71 & - & 0.54 & - & Schlueter et al., 2004 \\
\hline 39 Gene families & - & - & $0.57 \pm 0.05$ & $0.57 \pm 0.02$ & Pfeil et al., 2005 \\
\hline Lipoxygenases & 0.75 & $0.62^{c}$ & 0.46 & $0.45^{d}$ & This study \\
\hline
\end{tabular}

aThe median $\mathrm{Ks}$ value between paralogs represents polyploidy events.

bThe median Ks value between orthologs denotes taxon divergence between soybean and Medicago.

c, d The median Ks values between $\mathrm{Gm}-\mathrm{Mt}$ paralogs and $\mathrm{Gm}-\mathrm{Mt}$ orthologs are compared to rule out skewing estimates caused by differential substitution rates. Thus, the ancient polyploidy predates the taxon divergence. 


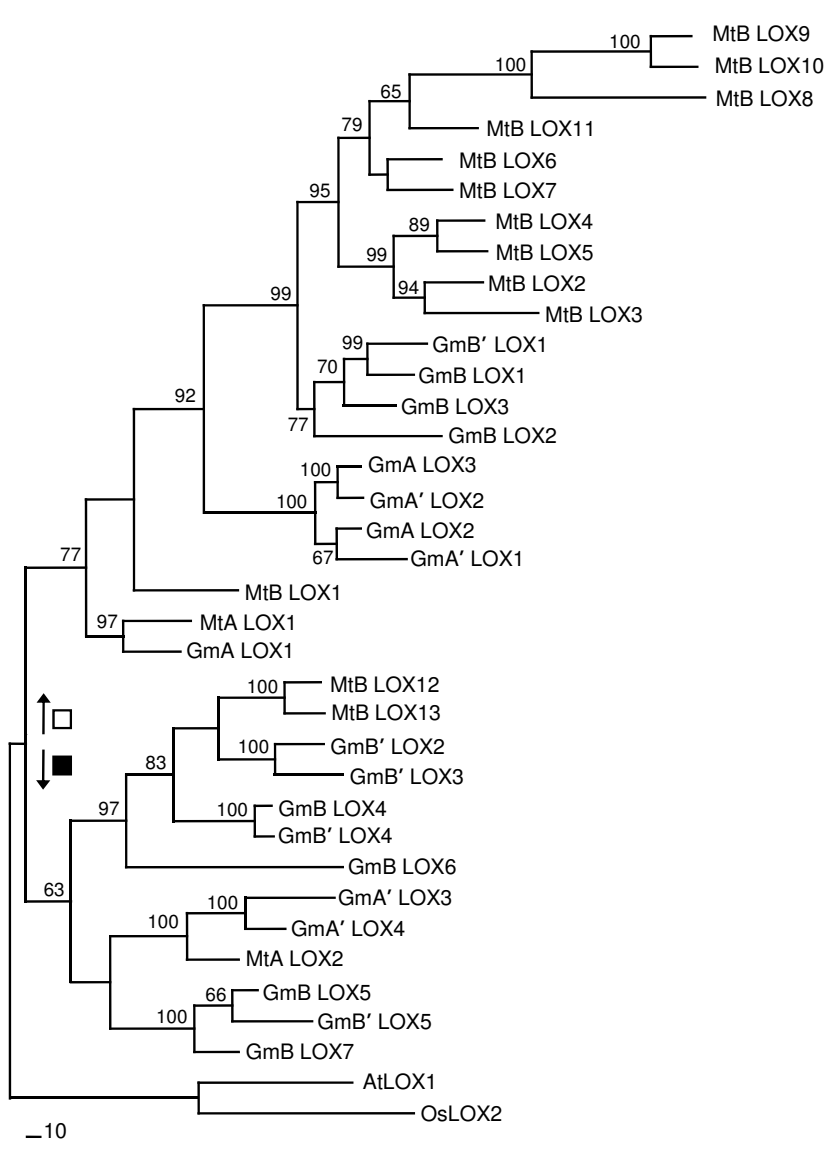

Figure 4

Phylogenetic analysis of $\mathbf{3 4}$ Lx proteins. A parsimony tree was generated using bootstrap analysis with I,000 replicates and branch swapping. Bootstrap values larger than 50 are denoted on each branch. The tree was rooted using Arabidopsis LOXI (AtLOXI) and rice LOXI (OsLOXI). Soybean and Medicago Lxs in homologous regions represented as black and white squares fall into two major clades. For convenience, each $L x$ gene is named according to its species, chromosomal region and physical order instead of its GenelD. The Lxs in GmA diverged earlier from $L x s$ in $G m B$ and $M t B$ and later $L x s$ in $G m B$ and MtB are separated, suggesting duplication before taxon divergence.

conclude whether Medicago and soybean, are allo- or autopolyploids. But it is clear that soybean and Medicago share both of the genome, rejecting the hypothesis of Medicago allopolyploid history after the Medicago-soybean split.

Expansion and functional divergence of the Lx gene family Phylogenetic analysis divided $34 L x$ genes identified in six $L x$ regions from soybean and Medicago into two clades (Figure 4). We expect two distinct $L x$ genes in the most recent common legume ancestor. This parsimony tree showed that the $L x$ genes in the $\mathrm{GmB}$ region were closer to the $L x$ genes in MtB than those of GmA, suggesting duplication prior to taxon divergence. After the split, tandem duplication of $L x$ genes occurred in $\mathrm{MtB}$, whereas the soybean $L x$ genes duplicated to $\mathrm{GmA} L x / \mathrm{GmA}^{\prime} L x$ and $\mathrm{GmB}$ $L x / \mathrm{GmB}^{\prime} L x$. This evolution of the $L x$ gene family in the homologous regions is diagrammatically represented in Figure 5 .

Duplicated genes have been reported to undergo nonfunctionalization, neo-functionalization, or sub-functionalization [44]. Among the $19 \mathrm{Lx}$ genes in the four soybean regions, nine were previously characterized and confirmed functional (see Additional file 2). In addition, the duplicated lipoxygenase genes had different activities at different $\mathrm{pH}$ values and different substrate specificities, suggesting differential functional specificities among lipoxygenase isoforms $[9,45]$. Moreover, the patterns of cellular and subcellular localization in pod walls were distinct among the isoforms, indicating independent functions [46]. Specialized isoforms are expected to improve the plant's flexibility to various environmental conditions.

Retention of multiple copies of Lxs in soybean, Medicago, and their common ancestor are reasonable from an evolutionary perspective because lipoxygenases confer various biotic and abiotic resistance traits to plants. Plant lipoxygenases have been reported to conferred resistance to stresses such as herbivores and wounding [47,48]. Also, clusters of genes related to resistance and disease response have been reported in soybean $[15,49]$. In grape (Vitis vinifera), the gene family encoding the grapevine phytoalexin is comprised of 43 genes, 20 of which were previously shown to be expressed [50]. Numerous $L x$ genes will increase protein or mRNA dosage, leading to resistance in plants. The beneficial effects of increased dosage of genes involved in defense or resistance has been reported in various studies: resistance to glyphosate in plants, protection against heavy metals in hamsters, and decreased susceptibility to HIV infection in humans [51-53]. This mechanism of gene family expansion and functional divergence of duplicated genes may also be relevant to understanding the evolution of other gene families.

A systematic approach is required for crop improvement and modification because most crops have more than one gene copy in their genomes. It is absolutely essential to investigate the number of loci of a particular gene of interest in the breeding of polyploid crops. Further understanding and insights into the paleopolyploid crop genome will lead to more efficient crop improvement and molecular breeding.

\section{Conclusion}

In this study, multiple $L x$ genes anchored in four soybean regions and two Medicago regions were analyzed at the sequence level. Differential evolutionary rates between soybean and Medicago were revealed among the six 
regions, with Medicago showing a greater synomymous substitution rate than soybean. This fact suggests that an optimized coalescence estimation is needed for each comparison: Gm-Gm, Gm-Mt or Mt-Mt. The four soybean Lx regions are comprised of two pairs of recently duplicated regions, and each pair is co-orthologous to one region in Medicago. These results support an ancient polyploidy in the common ancestor of soybean and Medicago, which preceded separation of the Hologalegina and phaseoloidmillettioid clades. Based on the tetrad soybean genome structure, four copies of duplicated genes or four homeologous regions in soybean are theoretically expected. Phylogenetic analysis showed that the $L x$ gene family basically expanded by whole genome duplication. Moreover, $L x$ genes underwent extensive tandem gene duplication.

\section{Methods}

Lipoxygenase BAC selection and mining of soybean super contigs

Three specific PCR primers were designed to select BAC clones that contained the target genes, $L x 1, L x 2$ and $L x 3$, based on GenBank acc. numbers L02795, L03211, and $\underline{\mathrm{U} 50081}$, respectively. The primer sequences were: $L x 1$ forward, 5'-TTA ATG CTT TCT TGG GCC CTA-3' and Lx1 reverse, 5'-CGC TCT CCC GTT CCA TTT CC-3'; $L x 2$ forward, 5'-GCT ATA AAT CAC GTT TCG TTA C-3' and Lx2 reverse, 5'-TAT GCC CTC CTC CTC TGT TC-3'; Lx3 forward, 5'-GTAGTGTTGGTGGGTTGCAAAGATG-3' and Lx3 reverse, 5'-GCA AAC AAA GTG GAT GCT TCC ATG-3'.

A pilot experiment was performed with G. max cultivar Williams 82, prior to BAC selection to optimize PCR conditions using a PTC-225-DNA gradient cycler from MJ research (Watertown, MA., USA). Williams 82 was used as a positive control during the selection procedure. The amplification reaction was $11 \mu \mathrm{l}$ in volume and contained $100 \mathrm{ng}$ of Williams 82 genomic DNA, 15 pmol of each forward and reverse primer, $10.0 \mathrm{mM}$ of dNTP mix, $1 \mu \mathrm{l}$ of $10 \times$ buffer, $6.9 \mu \mathrm{l}$ of dd- $\mathrm{H}_{2} \mathrm{O}$, and 0.2 unit of Taq DNA polymerase (Vivagen, Sungnam, Korea). The PCR conditions were $94^{\circ} \mathrm{C}$ for $2 \mathrm{~min}, 35$ cycles of $94^{\circ} \mathrm{C}$ for $30 \mathrm{sec}$, annealing temperature for $30 \mathrm{sec}, 72^{\circ} \mathrm{C}$ for $30 \mathrm{sec}$, and a final extension of $2 \mathrm{~min}$ at $72^{\circ} \mathrm{C}$.

The Williams 82 G. $\max$ BAC clone library [54] was PCRscreened using the same conditions as described above for the genomic DNA of Williams 82 . The final PCR screen was conducted with $0.2 \mu \mathrm{l}$ of the candidate BACs as a template from a working copy of the library.

Soybean super contigs (scaffolds) were identified by BLAST search with lipoxygenase genes against the soybean genome sequence produced by the Soybean Genome Project, DOE Joint Genome Institute http://www.phyto zome.com/soybean.

\section{Common ancestor}

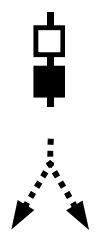

Duplicated common ancestor

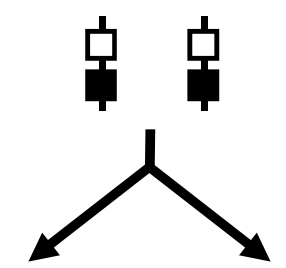

M. truncatula

G. $\max$

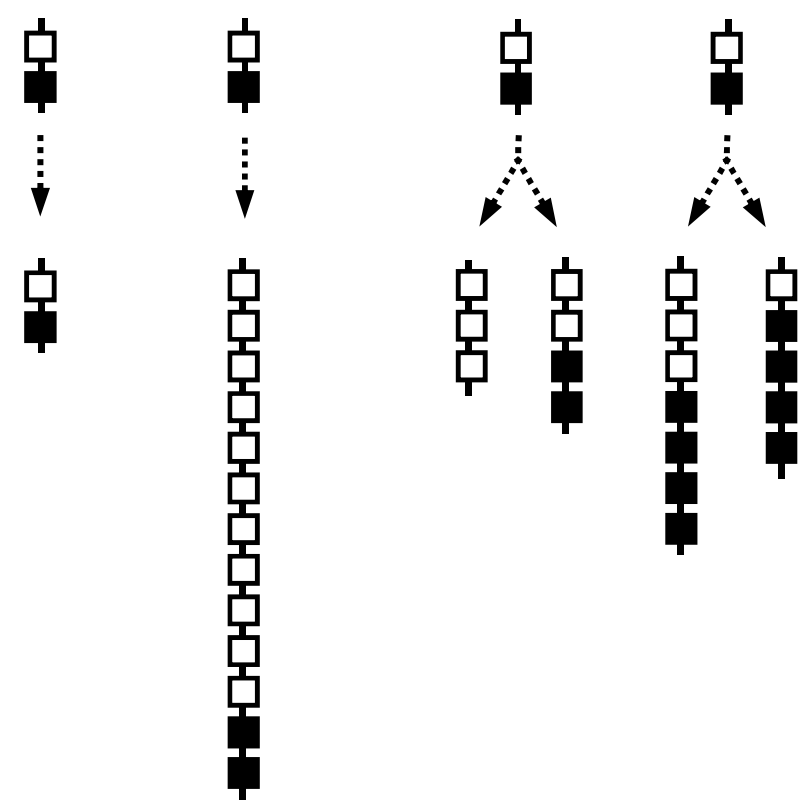

Figure 5

Expansion of the Lx gene family in soybean and Medicago homologous regions in relation to the evolutionary events in the six regions. No direct evidence of a recent polyploidy event in Medicago was detected; instead, tandem duplication of Lxs was observed in MtB. In soybean, two pairs of $L x$ regions generated by two rounds of polyploidy were identified and analyzed. Each pair of soybean regions is co-orthologous to the region in Medicago, suggesting co-orthologous regions produced by lineage-specific duplication in soybean.

\section{In silico mapping of BACs and super contigs}

Genetic markers for $L x 1, L x 2$ and $L x 3$ were defined on the consensus soybean genetic map (December, 2006; http:// soybase.org) and the sequences of the accessions from 
which the SNP-containing sequence tagged site was developed were compared with BAC clone sequences using BLAST2 http://www.ncbi.nlm.nih.gov/blast. Simple sequence repeat (SSR) markers in BAC clones and scaffolds were identified by BLAST search against genome survey sequence (GSS) records restricted to soybean SSRcontaining clones http://www.ncbi.nlm.nih.gov/blast. Thus, the genetic map positions of the selected BAC clones and scaffolds were determined by the loci of lipoxygenase and SSR markers.

\section{BAC sequencing and assembly}

Six BACs, gmw1-45b2 (EU028318), gmw1-91g6 (EU028319), gmw1-6b18 (EU028314), gmw1-9c4 (EU028315), gmw1-22a20 (EU028316) and gmw122f19 (EU028317), were sequenced using Genome Sequencer (GS)-FLX. Sequence data were assembled using Phred, Phrap, and Consed to diminish the number of contigs. The remaining gaps were closed by hybrid assembly [29], adding ABI-Sanger sequences from the end of the contigs.

\section{Sequence analysis and annotation}

Repetitive sequences were screened using RepeatMasker http://www.repeatmasker.org/.

Gene prediction of soybean and Medicago sequences was performed using FgeneSH on an Arabidopsis matrix, because the results were better suited for BLASTZ results than that of the Medicago matrix http://www.soft berry.com. Each predicted gene was annotated by BLASTP searches against UniProt. Syntenic regions in M. truncatula were detected using the BLASTN program with nucleotide collection restricted to $M$. truncatula. These syntenic regions were compared with Pipmaker [55], BLASTZ program, and visualized using SynBrowse http://www.syn browse.org and GBrowse http://www.gmod.org/ggb/ gbrowse.shtml.

\section{Nucleotide substitution rates, dating of duplication events, and phylogenetic analysis}

The Ks values between putative homologues were calculated using the PAML package [56]. Sequences of lipoxygenases in the six $L x$ regions of soybean and Medicago were compiled and aligned using ClustalX and sequence overhang at the 5'- and 3'-end of alignments were removed. A parsimony tree was generated using bootstrap analysis with 1,000 replicates and branch swapping in PAUP* 4.0 [57] and rooted with Arabidopsis and rice as out-groups.

\section{Authors' contributions}

JHS designed this study, selected BACs, produced the phylogenetic tree and analyzed the sequences. KV estimated Ks values and helped to design and draft the manuscript. DHK identified and sequenced BAC selections and annotated BACs. KDK mined syntenic regions in Medicago trun- catula, performed comparative genomics as well as Ks value estimation and mapped BACs and scaffolds in silico. BSC sequenced BACs and assembled their sequences. YEJ helped to design primers and select BAC clones. MYK helped to draft the manuscript. SHL helped to design this study as well as draft the manuscript. All authors have read and approved the final manuscript.

\section{Additional material}

\section{Additional File 1}

Assembly statistics of six BAC clones from GS-FLX. This data provided show the assembly statistics of six BAC clones from GS-FLX and the remaining gaps were closed by hybridization assemblies, adding ABISanger sequences amplified across the gaps.

Click here for file

[http://www.biomedcentral.com/content/supplementary/14712229-8-133-S1.doc]

\section{Additional File 2}

List of GenBank GeneIDs corresponding to soybean Lx genes with their phylogenetic relationships. A total of 13 soybean $\mathrm{Lx}$ genes were searched on NCBI and nine of them were included in GmA, GmA', GmB, and $G m B^{\prime}$

Click here for file

[http://www.biomedcentral.com/content/supplementary/14712229-8-133-S2.doc]

\section{Additional File 3}

Pairwise comparisons of Ks values between homologous genes. These Ks values of common genes among the six homologous regions show differential evolutionary rates between Medicago and soybean.

Click here for file

[http://www.biomedcentral.com/content/supplementary/1471-

2229-8-133-S3.doc]

\section{Additional File 4}

Descriptions of predicted genes based on UniRef results within the six $\mathrm{Lx}$ regions. This table provides descriptions of predicted genes and $\mathrm{Lx}$ genes are highlighted with green color.

Click here for file

[http://www.biomedcentral.com/content/supplementary/14712229-8-133-S4.doc]

\section{Acknowledgements}

This research was supported by a grant (grant no. 305005-4) for BAC clone seletion funded by the Agricultural R\&D Promotion Center, the Ministry of Food, Agriculture, Forestry and Fisheries, the Republic of Korea, in part by a grant (code no. CG3 I I) for genetic mapping from the Crop Functional Genomics Center of the $21^{\text {st }}$ Century Frontier Research Program funded by the Ministry of Education, Science and Technology (MEST), the Republic of Korea and a grant from the BioGreen 21 Project (code no. 20080401034010) for DNA sequencing, Rural Development Administration, the Republic of Korea. K. Van and K.D. Kim are the recipients of a fellowship from the BK2I program granted by MEST, the Republic of Korea.

\section{References}

I. Forster C, North H, Afzal N, Domoney C, Hornostaj A, Robinson DS, Casey R: Molecular analysis of a null mutant for pea 
(Pisum sativum L.) seed lipoxygenase-2. Plant Mol Biol 1999, 39(6): $1209-1220$

2. Gardner HW: 9-Hydroxy-traumatin, a new metabolite of the lipoxygenase pathway. Lipids 1998, 33(8):745-749.

3. Kolomiets MV, Chen H, Gladon RJ, Braun EJ, Hannapel DJ: A leaf lipoxygenase of potato induced specifically by pathogen infection. Plant Physiol 2000, I 24(3): I I I I- I 30.

4. Veronesi C, Rickauer M, Fournier J, Pouenat ML, Esquerre-Tugaye MT: Lipoxygenase gene expression in the tobacco-Phytophthora parasitica nicotianae interaction. Plant Physiol 1996, I I 2(3):997-1004

5. Bell E, Mullet JE: Characterization of an Arabidopsis lipoxygenase gene responsive to methyl jasmonate and wounding. Plant Physiol 1993, 103(4): I 133-I I37.

6. Davies CS, Nielsen SS, Nielsen NC: Flavor improvement of soybean preparations by genetic removal of lipoxygenase-2. Journal of the American Oil Chemists Society 1987, 64(10): I428-1433.

7. Kim MY, Ha BK, Jun TH, Hwang EY, Van K, Kuk YI, Lee SH: Single nucleotide polymorphism discovery and linkage mapping of lipoxygenase-2 gene (Lx2) in soybean. Euphytica 2004, I35(2):169-177.

8. Kitamura K, Kumagai T, Kikuchi A: Inheritance of lipoxygenase-2 and genetic-relationships among genes for lipoxygenase-I, lipoxygenase- 2 and lipoxygenase- 3 isozymes in soybean seeds. Japanese Journal of Breeding 1985, 35(4):413-420.

9. Kato T, Ohta H, Tanaka K, Shibata D: Appearance of new lipoxygenases in soybean cotyledons after germination and evidence for expression of a major new lipoxygenase gene. Plant Physiol 1992, 98(I):324-330.

10. Saravitz DM, Siedow JN: The differential expression of woundinducible lipoxygenase genes in soybean leaves. Plant Physiol 1996, I I O(I):287-299.

II. Kong $\mathrm{H}$, Landherr LL, Frohlich MW, Leebens-Mack J, Ma H, dePamphilis CW: Patterns of gene duplication in the plant SKP I gene family in angiosperms: evidence for multiple mechanisms of rapid gene birth. Plant J 2007, 50(5):873-885

12. Blanc G, Wolfe $\mathrm{KH}$ : Widespread paleopolyploidy in model plant species inferred from age distributions of duplicate genes. Plant Cell 2004, I6(7):1667-1678

13. Schlueter JA, Dixon P, Granger C, Grant D, Clark L, Doyle J], Shoemaker RC: Mining EST databases to resolve evolutionary events in major crop species. Genome/National Research Council Canada = Genome/Conseil national de recherches Canada 2004, 47(5):868-876.

14. Shoemaker RC, Polzin K, Labate J, Specht J, Brummer EC, Olson T, Young N, Concibido V, Wilcox J, Tamulonis JP, Kochert G, Boerma HR: Genome duplication in soybean (Glycine subgenus soja). Genetics 1996, 144(I):329-338

15. Schlueter JA, Scheffler BE, Schlueter SD, Shoemaker RC: Sequence conservation of homeologous bacterial artificial chromosomes and transcription of homeologous genes in soybean (Glycine max L. Merr.). Genetics 2006, 174(2): $1017-1028$.

16. Schlueter JA, Vasylenko-Sanders IF, Deshpande S, Yi J, Siegfried M, Roe BA, Schlueter SD, Scheffler BE, Shoemaker RC: The FAD2 gene family of soybean: insights into the structural and functional divergence of a paleopolyploid genome. Crop Science 2007, 47(Supplement_I): 14-26.

17. Schlueter JA, Lin JY, Schlueter SD, Vasylenko-Sanders IF, Deshpande S, Yi J, O'Bleness M, Roe BA, Nelson RT, Scheffler BE, Jackson SA Shoemaker RC: Gene duplication and paleopolyploidy in soybean and the implications for whole genome sequencing. BMC genomics 2007, 8:330.

18. Udall JA, Wendel JF: Polyploidy and crop improvement. Crop Science 2006:46.

19. Wendel JF: Genome evolution in polyploids. Plant Mol Biol 2000 42(I):225-249.

20. Thoquet P, Gherardi M, Journet EP, Kereszt A, Ane JM, Prosperi JM, Huguet T: The molecular genetic linkage map of the model legume Medicago truncatula: an essential tool for comparative legume genomics and the isolation of agronomically important genes. BMC Plant Biol 2002, 2:I.

21. Pfeil BE, Schlueter JA, Shoemaker RC, Doyle J]: Placing paleopolyploidy in relation to taxon divergence: a phylogenetic analysis in legumes using $\mathbf{3 9}$ gene families. Syst Biol 2005, 54(3):44|-454.
22. Cannon SB, Sterck L, Rombauts S, Sato S, Cheung F, Gouzy J, Wang X, Mudge J, Vasdewani J, Schiex T, SpannagI M, Monaghan E, Nicholson C, Humphray SJ, Schoof H, Mayer KF, Rogers J, Quetier F, Oldroyd GE, Debelle F, Cook DR, Retzel EF, Roe BA, Town CD, Tabata $S$, Peer $Y$ Van de, Young ND: Legume genome evolution viewed through the Medicago truncatula and Lotus japonicus genomes. Proc Natl Acad Sci USA 2006, 103(40): 14959-14964.

23. Zhang XC, Wu X, Findley S, Wan J, Libault M, Nguyen HT, Cannon SB, Stacey G: Molecular evolution of lysin motif-type receptorlike kinases in plants. Plant Physiol 2007, I 44(2):623-636.

24. Yan HH, Mudge J, Kim DJ, Larsen D, Shoemaker RC, Cook DR, Young ND: Estimates of conserved microsynteny among the genomes of Glycine max, Medicago truncatula and Arabidopsis thaliana. Theor Appl Genet 2003, I06(7): | 256-1265.

25. Cannon SB, McCombie WR, Sato S, Tabata S, Denny R, Palmer L, Katari M, Young ND, Stacey G: Evolution and microsynteny of the apyrase gene family in three legume genomes. Mol Genet Genomics 2003, 270(4):347-36I.

26. Mudge J, Cannon SB, Kalo P, Oldroyd GE, Roe BA, Town CD, Young ND: Highly syntenic regions in the genomes of soybean, Medicago truncatula, and Arabidopsis thaliana. BMC Plant Biol 2005, 5: 15

27. Schlueter JA, Scheffler BE, Jackson S, Shoemaker RC: Fractionation of Synteny in a Genomic Region Containing Tandemly Duplicated Genes across Glycine max, Medicago truncatula, and Arabidopsis thaliana. The Journal of heredity 2008

28. Reinprecht Y, Poysa VW, Yu K, Rajcan I, Ablett GR, Pauls KP: Seed and agronomic $Q T L$ in low linolenic acid, lipoxygenase-free soybean (Glycine max (L.) Merrill) germplasm. Genome/ National Research Council Canada $=$ Genome/Conseil national de recherches Canada 2006, 49(12):1510-1527.

29. Wicker T, Schlagenhauf E, Graner A, Close T], Keller B, Stein N: 454 sequencing put to the test using the complex genome of barley. BMC genomics 2006, 7:275.

30. Cregan PB, Kollipara KP, Xu SJ, Singh RJ, Fogarty SE, Hymowitz T: Primary trisomics and SSR markers as tools to associate chromosomes with linkage groups in soybean. Crop Science 200I, 4 I (4): I $262-1267$

31. Rector BG, All JN, Parrott WA, Boerma HR: Quantitative trait loci for antibiosis resistance to corn earworm in soybean. Crop Science 2000, 40(I):233-238.

32. Specht JE, Chase K, Macrander M, Graef GL, Chung J, Markwell JP Germann M, Orf JH, Lark KG: Soybean response to water: A QTL analysis of drought tolerance. Crop Science 200I, $4 I(2): 493-509$.

33. Terry LI, Chase K, Jarvik T, Orf J, Mansur L, Lark KG: Soybean quantitative trait loci for resistance to insects. Crop Science 2000, 40(2):375-382.

34. Wang D, Graef GL, Procopiuk AM, Diers BW: Identification of putative QTL that underlie yield in interspecific soybean backcross populations. Theor Appl Genet 2004, 108(3):458-467.

35. Maughan PJ, Maroof MAS, Buss GR: Identification of quantitative trait loci controlling sucrose content in soybean (Glycine max). Molecular Breeding 2000, 6(I): 105-III

36. Diers BW, Keim P, Fehr WR, Shoemaker RC: RFLP analysis of soybean seed protein and oil content. Theor Appl Genet 1992, 83(5):608-6/2

37. Mansur LM, Lark KG, Kross $\mathrm{H}$, Oliveira $\mathrm{A}$ : Interval mapping of quantitative trait loci for reproductive, morphological, and seed traits of soybean (Glycine max L.). Theor Appl Genet 1993, 86(8):907-9|3.

38. Heer JA, Knap HT, Mahalingam R, Shipe ER, Arelli PR, Matthews BF: Molecular markers for resistance to Heterodera glycines in advanced soybean germplasm. Molecular Breeding 1998, 4(4):359-367.

39. Triwitayakorn K, Njiti VN, Iqbal MJ, Yaegashi S, Town C, Lightfoot DA: Genomic analysis of a region encompassing QRfsI and QRfs2: genes that underlie soybean resistance to sudden death syndrome. Genome/National Research Council Canada = Genome/Conseil national de recherches Canada 2005, 48(I): I25-I 38.

40. Shoemaker RC, Schlueter J, Doyle JJ: Paleopolyploidy and gene duplication in soybean and other legumes. Curr Opin Plant Biol 2006, 9(2): 104-109.

4I. Koonin EV: Orthologs, paralogs, and evolutionary genomics. Annu Rev Genet 2005, 39:309-338. 
42. Lavin M, Herendeen PS, Wojciechowski MF: Evolutionary rates analysis of Leguminosae implicates a rapid diversification of lineages during the tertiary. Syst Biol 2005, 54(4):575-594.

43. Van K, Kim DH, Cai CM, Kim MY, Shin JH, Graham MA, Shoemaker RC, Choi BS, Yang TJ, Lee SH: Sequence Level Analysis of Recently Duplicated Regions in Soybean [Glycine max (L.) Merr.] Genome. DNA Res 2008, 15(2):93-102.

44. Lynch M, Conery JS: The evolutionary fate and consequences of duplicate genes. Science 2000, 290(5494): II5I-II55.

45. Fuller MA, Weichert H, Fischer AM, Feussner I, Grimes HD: Activity of soybean lipoxygenase isoforms against esterified fatty acids indicates functional specificity. Arch Biochem Biophys 200I, 388(1): $146-154$.

46. Dubbs WE, Grimes HD: Specific lipoxygenase isoforms accumulate in distinct regions of soybean pod walls and mark a unique cell layer. Plant Physiol 2000, I 23(4): 1269-1280.

47. Wang R, Shen W, Liu L, Jiang L, Liu Y, Su N, Wan J: A novel lipoxygenase gene from developing rice seeds confers dual position specificity and responds to wounding and insect attack. Plant Mol Biol 2008, 66(4):40I-4I4.

48. Wu J, Wang L, Baldwin IT: Methyl jasmonate-elicited herbivore resistance: does MeJA function as a signal without being hydrolyzed to JA? Planta 2008, 227(5): I|61-I|68.

49. Graham MA, Marek LF, Shoemaker RC: Organization, expression and evolution of a disease resistance gene cluster in soybean. Genetics 2002, I62(4): | 96|-1977.

50. Jaillon O, Aury JM, Noel B, Policriti A, Clepet C, Casagrande A, Choisne N, Aubourg S, Vitulo N, Jubin C, Vezzi A, Legeai F, Hugueney P, Dasilva C, Horner D, Mica E, Jublot D, Poulain J, Bruyere C, Billault A, Segurens B, Gouyvenoux M, Ugarte E, Cattonaro F, Anthouard V, Vico V, Del Fabbro C, Alaux M, Di Gaspero G, Dumas V, Felice N, Paillard S, Juman I, Moroldo M, Scalabrin S, Canaguier A, Le Clainche I, Malacrida G, Durand E, Pesole G, Laucou V, Chatelet P, Merdinoglu D, Delledonne M, Pezzotti M, Lecharny A, Scarpelli C, Artiguenave F, Pe ME, Valle G, Morgante M, Caboche M, Adam-Blondon AF, Weissenbach J, Quetier F, Wincker P, French-Italian Public Consortium for Grapevine Genome Characterization: The grapevine genome sequence suggests ancestral hexaploidization in major angiosperm phyla. Nature 2007, 449(716I):463-467.

5I. Gonzalez E, Kulkarni H, Bolivar H, Mangano A, Sanchez R, Catano G, Nibbs RJ, Freedman BI, Quinones MP, Bamshad MJ, Murthy KK, Rovin $\mathrm{BH}$, Bradley W, Clark RA, Anderson SA, O'connell RJ, Agan BK, Ahuja SS, Bologna R, Sen L, Dolan MJ, Ahuja SK: The influence of CCL3LI gene-containing segmental duplications on HIV-I/ AIDS susceptibility. Science 2005, 307(57 | 4): |434-|440.

52. Palmiter RD: Protection against zinc toxicity by metallothionein and zinc transporter I. Proc Natl Acad Sci USA 2004, I0I(14):49|8-4923.

53. Widholm JM, Chinnala AR, Ryu JH, Song HS, Eggett T, Brotherton JE: Glyphosate selection of gene amplification in suspension cultures of 3 plant species. Physiol Plant 200 I, I I 2(4):540-545.

54. Marek LF, Shoemaker RC: BAC contig development by fingerprint analysis in soybean. Genome/National Research Council Canada $=$ Genome/Conseil national de recherches Canada 1997, 40:420-427.

55. Schwartz S, Zhang Z, Frazer KA, Smit A, Riemer C, Bouck J, Gibbs R, Hardison R, Miller W: PipMaker-a web server for aligning two genomic DNA sequences. Genome Res 2000, I0(4):577-586

56. Yang Z: PAML: a program package for phylogenetic analysis by maximum likelihood. Comput Appl Biosci 1997, I3(5):555-556.

57. Swofford DL: PAUP*. Phylogenetic analysis using parsimony (*and Other Methods). In Version 4 Sinauer Associates, Sunderland, Massachusetts; 2003.

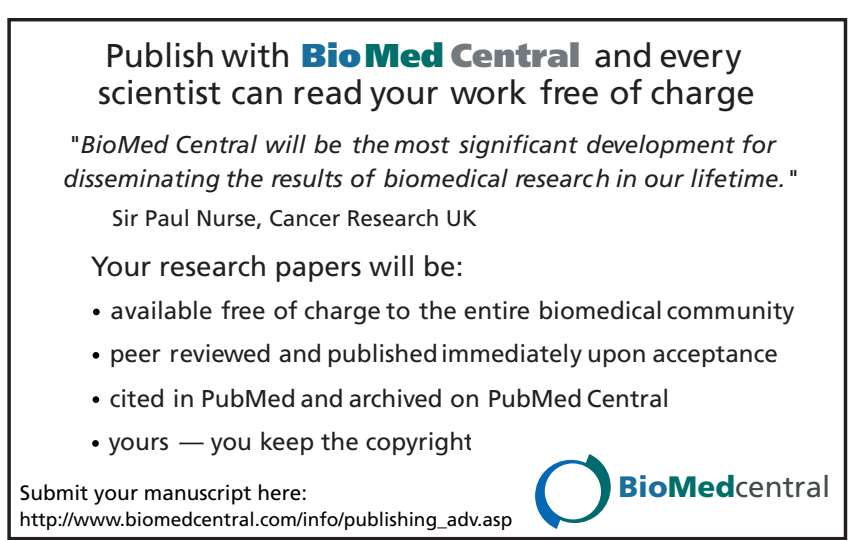

